\title{
THE POTENTIAL OF LIGHT LASER SCANNERS DEVELOPED FOR UNMANNED AERIAL VEHICLES - THE REVIEW AND ACCURACY
}

\author{
M. Pilarska ${ }^{\text {a }}$,W. Ostrowski ${ }^{\text {a }}$, K. Bakuła ${ }^{\text {a }}$, K. Górski ${ }^{\text {a }}$ Z. Kurczyński ${ }^{\text {a }}$ \\ ${ }^{a}$ Warsaw University of Technology, Faculty of Geodesy and Cartography, Department of Photogrammetry, Remote Sensing and \\ Spatial Information Systems, Warsaw, Poland - pilarska-magdalena@wp.pl, (w.ostrowski, k.bakula)@ gik.pw.edu.pl,
} gor.konrad@gmail.com, kurczynski@wp.pl

\section{Commission I}

KEY WORDS: UAV, laser scanning, light scanners, accuracy, review, LiDAR

\begin{abstract}
:
Modern photogrammetry and remote sensing have found small Unmanned Aerial Vehicles (UAVs) to be a valuable source of data in various branches of science and industry (e.g., agriculture, cultural heritage). Recently, the growing role of laser scanning in the application of UAVs has also been observed. Laser scanners dedicated to UAVs consist of four basic components: a laser scanner (LiDAR), an Inertial Measurement Unit (IMU), a Global Navigation Satellite System (GNSS) receiver and an on-board computer. The producers of the system provide users with detailed descriptions of the accuracies separately for each component. However, the final measurement accuracy is not given. This paper reviews state-of-the-art of laser scanners developed specifically for use on a UAV, presenting an overview of several constructions that are available nowadays. The second part of the paper is focussed on analysing the influence of the sensor accuracies on the final measurement accuracy. Mathematical models developed for Airborne Laser Scanning (ALS) accuracy analyses are used to estimate the theoretical accuracies of different scanners with conditions typical for UAV missions. Finally, the theoretical results derived from the mathematical simulations are compared with an experimental use case.
\end{abstract}

\section{INTRODUCTION}

The market for laser scanners applicable in Unmanned Aerial Vehicles (UAVs), robotics and autonomous driving is growing rapidly. It offers a variety of solutions, creating new possibilities for research and commercial sectors. Some of these scanners are designed for use on unmanned platforms, and others typically used as Terrestrial Laser Scanner (TLS) are just compact enough to mount on a UAV and be used as a lightweight laser scanner LiDAR. A challenging task over the past few years was to build a complete LiDAR scanning system based on light UAV platforms. The main problem that had to be solved was weight reduction. The weight of a platform is crucial for several important reasons. The first is the flight time, which shortens significantly with an increase in weight. This severely affects the efficiency of the scanning platform. The second matter is legal restrictions and legislation, which differ in every part of the globe. Consequently, an unmanned platform should be light and versatile. As a result, a strong need for building light, compact and accurate laser scanning modules can be observed. This issue is strictly related to two main components of the system: a laser scanner and a navigation module (GNSS/INS). Today, a significant step forwards can be observed. More and more lightweight laser scanners and ready-to-use solutions are being offered by manufacturers all over the world. This seems to be an answer to increasing market demands. Recent papers (Petrie, 2013; Colomina \& Molina, 2014; Starek \& Jung, 2015) concerning the development of light UAV imaging and laser scanning systems have revealed high potential and fast growth in this market sector. As a result, new devices and ready-to-fly solutions can be observed today. Some of these seem to be gamechanging and leading the market at the moment. Still, minimising some payloads (e.g., optical, hyperspectral or thermal cameras) is challenging for producers. This trend is also noticeable considering LiDAR technology, even bathymetric LiDAR (Mandlburger et al., 2016). Nowadays, laser scanners are also much lighter and cheaper. Such trends can be related to the development of technology, but they can (though not always) result in lower measurement accuracy. This paper focuses on the lightest scanning systems available and small UAVs flying at low altitudes.

The LiDAR and navigational modules mounted on a UAV are different from those mounted on airplanes for aerial data acquisition. For a small UAV, the weight of the payload is one of the most crucial elements that limit the usage of high-quality components, which directly influences the measurement accuracy. Navigational modules-IMU and GNSS receiversare lighter and smaller than those mounted on airplanes. In the last few years, the development of lightweight navigation sensors has also been noticed. Since 2014, companies like Trimble (with APX-15), SBG (with the Eclipse series) and NovAtel (with STIM300 and the SPAN series) have presented new solutions for the precise positioning of UAVs. In general, this new type of integrated sensor contains a double-frequency GNSS receiver and a new generation of tactical-grade MEMS IMUs, which make it possible to precisely determine the trajectory, and the weight of most of these sensors is not problematic because it is less than 100 grams.

In this article, the main factors and their influence on the final results are also presented. Understanding how they can affect the measurement accuracy plays an important role. Proper system calibration and time synchronisation between the components, as well as acquaintance with position errors, attitude errors and range measurement errors, are crucial for further UAV-sourced data processing and products' accuracy. The influence of these factors can be estimated using a mathematical model. The model 
is a function of the position error, orientation error, range measurement error, bore-sight orientation error, scanner error and lever-arm error between the scanner and the IMU. The influence on the $\mathrm{x}, \mathrm{y}$ and $\mathrm{z}$ coordinates' accuracy is additionally graphically presented. Besides the aforementioned error sources, more factors can affect the measurement accuracy; nevertheless, it is not possible to estimate their influence using the mathematical model. Included in this group is, for example, the vibration of the mechanical components of the sensor ( $\mathrm{Li}$ et al., 2015). The temperature of the sensor may also play an important role. Specifically, the internal laser temperature stability, which was examined by Glennie et al. (2016), correlates with range measurement errors. As a result, many LiDAR sensors contain a range correction, whose value depends on the laser operating temperature.

The following three sections present the potential of light laser scanners developed for unmanned aerial vehicles. The second section describes existing solutions for UAVs Laser Scanning (ULS), with distinction between laser scanners, GNSS and IMU units. The third section explains the source of errors that affect the final accuracy of the point cloud delivered by LiDAR systems mounted on UAVs and calculates the theoretical results using the mathematical model developed by Vosselman and Maas (2010). In this section the influence of errors is also visualised and existing producers' solutions are examined. In the discussion, the model of errors tested is compared with the results of the authors presenting their accuracy with the described scanning systems. Final conclusions are drawn in the last chapter.

\section{LIGHT LASER SCANNERS FOR UNMANNED AERIAL VEHICLES}

The basic components of the LiDAR scanning system are the emitter and the receiver of the laser beam (scanner) and the sensors responsible for the orientation of the scanner in the space associated with the determination of the position and inclination angles. This section presents the basic issues related to these components and existing solutions on the market, as well presents the current state-of-the-art in this branch of LiDAR technology for the reader.

\subsection{Laser scanners}

To date, not many papers have categorised lightweight laser scanners. To put current solutions in order, Petrie (2013) proposed to distinguish between them by scanning device type. Starek and Jung (2015) attributed scanners to the type (mainly size and weight) of UAV on which they are to be mounted. According to Petrie (2013), four main groups can be specified: (1) simple scanners, (2) multilayer laser scanners, (3) multiple spinning laser scanners and (4) terrestrial 3D laser scanners. Table 2 in appendix shows example UAV LiDAR systems (ULS) specifications that are the most important for their comparison.

Simple scanners (1) use laser rangefinders and rotating mirrors to generate a 2D scanning plane. This type of device was primarily dedicated to robotics and automation. However, their ranging possibilities were successfully adapted to surveying. Popular representatives of this group are Hokuyo devices. These rangefinders are used in the Sabre Sky-3D S80 scanner. But they are not the only group among simple scanners. In 2014, Riegl presented its new, first-in-company-history, survey-grade, UAV -dedicated laser scanner: the VUX-UAV1. It is now considered a milestone in the UAV scanners market. Since it is Riegl's flagship UAV product, it is also offered as a complete scanning system combined with RiCOPTER, the company's multirotor platform.

The second group is multilayer scanners (2), which utilise a number of scanning planes as well as measure distances and angles simultaneously for every plane. Their development and growth are mostly due to the automotive industry since they have been used for anti-collision purposes. A popular representative is Ibeo LUX, used by the YellowScan Mapper scanner, which is the only commercial solution known to the authors using this type of scanner for measuring and mapping purposes.

Multiple spinning laser scanners (3), which are also the most popular, use dozens of individual lasers. The best-known producer is Velodyne, with its 16- and 32-laser solutions. These types of scanning devices have also been developed for the automotive industry, where their primary application is measuring the neighbourhood of self-driving cars. Nowadays, these scanners also have wide applications in mobile mapping systems. The majority of available turn-key UAV LiDAR solutions is based on multiple spinning laser scanners. The most popular devices are Velodyne's HDL-32 and VLP-16. VLP-16 was presented by the manufacturer in Q3 2014 and priced at $\$ 8000$, which was considered very attractive. A significant improvement was a lightweight version of the popular VLP-16, the PUCK Lite scanner, presented in Q1 2016. The Lite version has identical performance to the prior VLP-16, but the weights are respectively $530 \mathrm{~g}$ and $830 \mathrm{~g}$. Also, in 2015, a new company related to the automotive industry, Quanergy, released its new M8 scanner. This product might also be adapted to be a 3D mapping sensor. The M8 scanner generates only 8 laser beams, which is not very many compared with Velodyne's products. However, its range, accuracy, weight $(800 \mathrm{~g})$ and price make it a very competitive alternative.

The fourth group (4) of available solutions are scanning systems based on terrestrial 3D laser scanners. Both of the solutions of this type known to the authors have already been mentioned in Petrie (2013); both utilise small terrestrial laser scanners developed by FARO. Only one of them is a turn-key solution, which was developed by Sabre: The Skypod Sky-3D S120 can be used with any UAV capable of carrying this almost 8-kg payload.

However, because the aim of this paper is to compare parameters of turn-key solutions available on the market, this issue can be introduced in a slightly different way. If we do not take into consideration the combined but complete UAV scanning systems, then available solutions can be divided in a slightly different way: (1) lightweight and miniaturised, UAV-applicable, airborne LiDAR systems; (2) terrestrial laser scanner-based systems; and (3) lightweight, UAV-dedicated scanning systems based on multilayer and multiple spinning laser scanners. Table 1 presents light UAV scanners specifications. Obviously, solutions proposed in first two groups are much different than those in the third one. Group (1) and (2) scanners are generally based on survey-grade components and simply modified and adopted to fit the UAV platform. Their measurement accuracy as well as their price are just too high to honestly compare these systems with solutions in group (3). Hence, in the following parts of this paper, group (3) systems will be discussed. 


\begin{tabular}{|c|c|c|c|c|}
\hline & 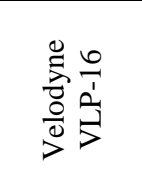 & 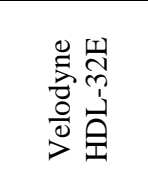 & 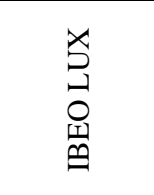 & $\begin{array}{l}\sum_{0}^{\infty} \\
\text { d. } \\
\vec{\Xi} \\
\bar{\Xi} \\
\tilde{\Xi}\end{array}$ \\
\hline Range $[\mathrm{m}]$ & 100 & 100 & 200 & 150 \\
\hline $\begin{array}{l}\text { Number of } \\
\text { layers }\end{array}$ & 16 & 32 & $4(2)$ & 8 \\
\hline $\begin{array}{l}\text { Horizontal } \\
\text { FOV }\end{array}$ & $360^{\circ}$ & $360^{\circ}$ & $85^{\circ}\left(100^{\circ}\right)$ & $360^{\circ}$ \\
\hline $\begin{array}{l}\text { Angular } \\
\text { resolution }\end{array}$ & $0.1^{\circ}-0.4^{\circ}$ & $0.16^{\circ}-0.4^{\circ}$ & $0.12^{\circ}-1^{\circ}$ & $\begin{array}{c}0.03^{\circ}- \\
0.2^{\circ}\end{array}$ \\
\hline $\begin{array}{l}\text { Vertical } \\
\text { FOV }\end{array}$ & $30^{\circ}$ & $40^{\circ}$ & $12.8^{\circ}$ & $20^{\circ}$ \\
\hline $\begin{array}{l}\text { Angle } \\
\text { between } \\
\text { beams }\end{array}$ & $2^{\circ}$ & $1.33^{\circ}$ & $3.2^{\circ}$ & $2.5^{\circ}$ \\
\hline $\begin{array}{l}\text { Range } \\
\text { accuracy }\end{array}$ & $3 \mathrm{~cm}$ & $2 \mathrm{~cm}$ & $10 \mathrm{~cm}$ & $5 \mathrm{~cm}$ \\
\hline $\begin{array}{l}\text { Range } \\
\text { precision }\end{array}$ & $2 \mathrm{~mm}$ & $2 \mathrm{~mm}$ & $4 \mathrm{~cm}$ & - \\
\hline $\begin{array}{l}\text { Dimensions } \\
{[\mathrm{mm}]}\end{array}$ & $103 \times 72$ & $85 \times 144$ & $\begin{array}{c}85 \times 128 \times \\
93\end{array}$ & $97 \times 87$ \\
\hline Weight & $830 \mathrm{~g}$ & $1050 \mathrm{~g}$ & $1000 \mathrm{~g}$ & $800 \mathrm{~g}$ \\
\hline
\end{tabular}

Table 1. Light UAV scanners' specifications

\subsection{Positioning systems for UAV}

One of the major differences between acquiring laser scanning and image data sets is the strict requirement for a precise trajectory for the LiDAR data set. Whereas for imagery data exterior orientation measurements are something additional, providing the possibility to reduce fieldwork or improve accuracy results, in the case of LiDAR these measurements are completely useless without the precise positioning described by the trajectory. Today, many lightweight GNSS/INS solutions can be found. Strictly specialised companies have been able to create light, tiny and relatively cheap integrated modules dedicated to robotics and UAVs. Moreover, their products ensure accuracy and precision high enough to make direct georeferencing of LiDAR possible. This class is called MEMS modules. The main advantage of these devices is their simple integration with any mobile platform (e.g., UAVs). These modules combine two crucial types of information: position (3D) and angle (3 axes). It is now possible to have these parameters with a module weighing less than 100 grams. One popular example is the Trimble APX15 , an integrated GNSS/INS module.

Other modern solutions are described in Table 3 in appendix to show their possibilities. All of them are part of available commercial systems and academic research platforms as well. These solutions are equipped with different GNSS types (single or dual antenna with one or two frequencies, using only Global Positioning System - GPS or with the inclusion of other GNSS systems) and various GNSS techniques (Differential Global Positioning System-DGPS, Precise Point Positioning-PPP, Real-Time Kinematic-RTK). Their angular accuracy (roll, pitch and heading) as well as gyroscope accuracy differ widely and depend mainly on the type of sensor. The last difference between these solutions shown in Table 3 in appendix is weight.

\section{ERROR SOURCES AND THEIR INFLUENCE ON MEASUREMENT ACCURACY}

At present, only a few publications are related to the accuracy of light laser scanners mounted on UAVs (Glennie et al., 2016; Mitteta et al., 2016). Moreover, only a few articles regarding the measurement accuracy using experimental data from light laser scanners can be found (Bakuła et al., 2016; Jozkow et al., 2016), and because of the different laser scanners used in the experiments, the results are on divergent levels. On the contrary, many publications refer to the accuracy of airborne laser scanning, but airborne scanning differs from UAV scanning; therefore, in this article the theoretical measurement accuracy of light laser scanners is analysed.

\subsection{Error sources}

The modules described in the previous part of this article are characterised by determined accuracy. Many publications about the error sources in airborne laser scanning systems have already been written (Wang et al., 2008; Shaer et al., 2007). The construction of light laser scanners mounted on UAVs is similar to the airborne ones; therefore, the same error sources are analysed in this paper. On the other hand, airborne laser scanning differs from UAV laser scanning regarding both modules' weight and flying altitude; therefore, it is worth analysing the final measurement accuracy of light laser scanners mounted on UAVs. Generally speaking, two types of errors can be distinguished: angular errors and linear errors. Angular errors are related to the orientation between the scanner components, while linear errors are connected with the measurement accuracy of linear elements (e.g., range). In this part of the article the error classification based on Vosselman and Mass (2010) is presented.

\section{GPS/INS positioning and orientation errors $R_{b}^{b^{\prime}}(\Delta r \Delta p \Delta y)$}

GPS/INS positioning errors are caused by various factors. The basic factor is a proper satellite configuration, which implies a number of observable satellites, baseline geometry, etc. The other accuracy elements are related to the multipath, clock biases and atmospheric conditions, such as differential troposphere effects or ionosphere delay (Wang et al., 2008). GPS/INS orientation errors of roll, pitch and yaw $(\Delta r \Delta p \Delta y)$ are determined for each axis individually. The INS accuracy does not stay constant for a long time; rather, it has a tendency to decrease with time. Therefore, the integration of GPS and INS, mostly using the Kalman filter, leads to the correction of the INS orientation parameters, and as a result, the navigational accuracy increases (Sharma et al., 2011).

\section{Bore-sight orientation errors $R_{s^{\prime}}^{b}(\Delta \omega \Delta \varphi \Delta \kappa)$}

Bore-sight errors correlate with an imprecisely orientated IMU component and the laser scanning plane. In practice, the error value depends on the system but can reach even degrees. Boresight effects due to roll errors are visible on the cross section when there are differences in a plane that was registered twice from crossed strips flown at different heights. Errors due to pitch and yaw cannot be noticed from the profile as easily as those due to roll, which makes cross sectioning an inappropriate method to estimate the total bore-sight errors (Skaloud, Lichti, 2006).

\section{Lever-arm errors $\Delta x_{b}^{s}$}

Lever-arm errors are connected to misalignment between sensors. If the lever-arm values are small (up to one meter), their influence on the measurement is insignificant. However, the lever arm between the body frame and the GPS antenna can reach several meters for airborne laser scanners; in such cases the accuracy of the lever-arm estimation needs to be considered. 


\section{$\underline{\text { Ranging } \Delta \rho \text { and angular errors } \Delta \theta}$}

Range accuracy depends on many factors, which complicates the estimation of the error value. For pulse systems such factors as time interval measurement and frequency instability can directly influence ranging errors (Baltsavias, 1999). Range measurements should also be corrected regarding the atmospheric refraction. Angular error is also influenced by many elements. One of the most important is laser-beam divergence, which results in an incorrectly measured point position. A second aspect is related to the mechanism of the scanner. High accelerations can result in a mirror position change. If the angular position between the scan mirror and the encoder changes, the value is incorrectly registered by the encoder.

Misalignment between the angular encoder and the scanning plane $\Delta \eta$

If the encoder is not installed perpendicular to the rotation angle of the mirror, a misalignment error occurs. As a result, there is an impression that the spacing between the encoder bins seems to change with the incidence angle. This error should be calibrated and also separated from the bore-sight error.

\subsection{Mathematical model}

The modules producers provide users with a detailed description of the accuracies for every component separately. However, the expected measurement accuracy is not determined. Therefore, it is difficult to estimate the final measurement accuracy and compare the systems with each other. In this part of the article, the influence of the modules' accuracies on the final measurement accuracy is analysed. The point coordinates from a laser scanning observation as well as the error of the observation can be calculated based on mathematical formulas.

Vosselman and Maas (2010) proposed an error model in which the errors described above are included. In the analysis of ranging errors, angular errors, GNSS/INS positioning and orientation errors (are considered. Bore-sight errors and lever-arm errors are not taken into account because their values are determined during calibration. If the calibration is done properly, the influence on lever-arm errors is negligible, especially for the UAV technology, where the lever-arm values are smaller than for airborne laser scanners.

$$
\begin{aligned}
x_{p}^{e}= & x_{b}^{e}+\Delta \hat{x}_{b}^{e}+\mathrm{R}_{l}^{e} \mathrm{R}_{b^{\prime}}^{l}(\hat{r} \hat{p} \hat{y}) \mathrm{R}_{b}^{b^{\prime}}(\Delta r \Delta p \Delta y) \mathrm{R}_{s^{\prime}}^{b}(\Delta \omega \Delta \varphi \Delta \kappa) \mathrm{T}_{s}^{s^{\prime}}(\hat{\omega} \hat{\varphi} \hat{\kappa}) \\
& \times\left(\begin{array}{c}
-(\hat{\rho}+\Delta \rho) \sin (\Delta \eta) \\
(\hat{\rho}+\Delta \rho) \sin (\hat{\theta}+\Delta \theta) \cos (\Delta \eta)+\hat{x}_{b}^{s}+\Delta x_{b}^{s} \\
(\hat{\rho}+\Delta \rho) \cos (\hat{\theta}+\Delta \theta) \cos (\Delta \eta)
\end{array}\right)
\end{aligned}
$$

where

$$
\begin{aligned}
& \mathrm{x}_{\mathrm{b}}^{\mathrm{e}} \text {-GPS/INS positioning errors } \\
& \mathrm{R}_{\mathrm{b}}^{\mathrm{b}^{\prime}}(\Delta \mathrm{r} \Delta \mathrm{p} \Delta \mathrm{y}) \text {-GPS/INS orientation errors } \\
& \mathrm{R}_{\mathrm{s}^{\prime}}^{\mathrm{b}}(\Delta \omega \Delta \varphi \Delta \kappa) \text {-bore-sight orientation errors } \\
& \mathrm{T}_{\mathrm{s}}^{\mathrm{s}^{\prime}}(\widehat{\omega} \hat{\varphi} \hat{\kappa}) \text {-part of the installation matrix } \\
& \Delta \mathrm{x}_{\mathrm{b}}^{\mathrm{s}} \text {-lever-arm error } \\
& \Delta \rho \text {-ranging error } \\
& \Delta \theta \text { - encoder/scanner error }
\end{aligned}
$$

First, the influence of the orientation errors and scanner errors on the $\mathrm{x}, \mathrm{y}$ and $\mathrm{z}$ coordinates is presented. The assumed flight height is $40 \mathrm{~m}$ and the maximum incidence angle is 60 degrees because, based on Glennie and Lichti (2010), when the incidence angle is larger than 65 degrees, the error values grow rapidly. Additionally, based on the same publication, the incidence angle accuracy (dTheta) is assumed to be equal to 0.03 degrees. The assumed roll $(d R o l l)$ and pitch (dPitch) errors are 0.02 degrees, and the yaw error (dYaw) equals 0.1 degrees. The range value changes with the incidence angle, so in the mathematical model a measurement error for the scanned flat surface is considered. The range error $(d R o)$ is $3 \mathrm{~cm}$.
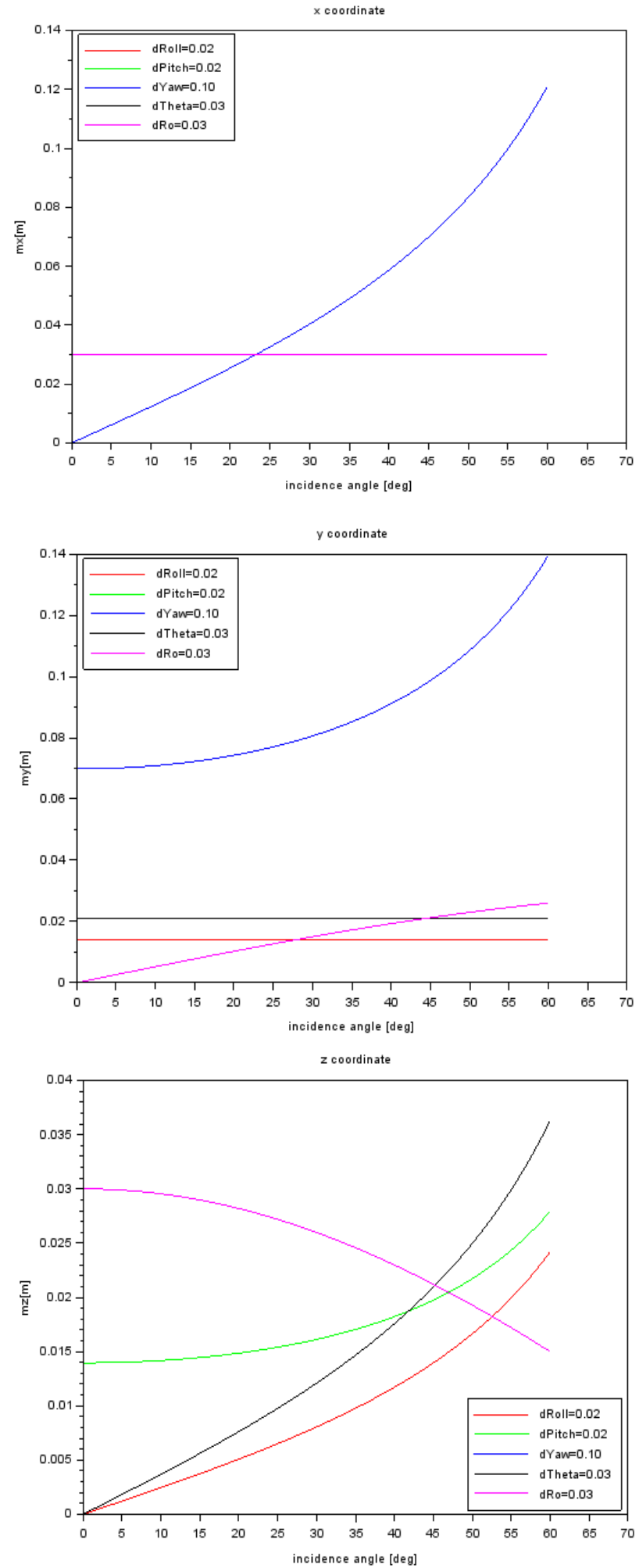

Figure 1. Effect of roll, pitch, yaw, theta and range errors on the $\mathrm{x}, \mathrm{y}$ and $\mathrm{z}$ accuracies relating to the incidence angle value 
In the graph in Fig. 1, the influences of the roll, pitch, yaw (heading), range and incidence angle errors on the $\mathrm{x}, \mathrm{y}$ and $\mathrm{z}$ coordinates are presented. The first graph shows the $\mathrm{x}$ coordinate error, considering every factor separately. Incidence angle, roll and pitch do not influence the coordinate's accuracy. Only range and yaw errors affect the $\mathrm{x}$ accuracy. The range influence is constant and does not depend on the incidence angle value, while the yaw impact is noticeable and grows fast. The y coordinate is influenced by more factors; only the pitch influence equals 0 . Incidence angle and roll have a constant impact, similar to the range influence for the $\mathrm{x}$ coordinate. For the $\mathrm{y}$ coordinate, range influence grows with the incidence angle value. Still, the biggest influence is represented by yaw (heading). For the assumed yaw error, the y error can reach up to $15 \mathrm{~cm}$ for an incidence angle equal to 60 degrees. Analysing the $\mathrm{z}$ coordinate accuracy, it is noticeable that all factors besides the yaw angle affect the coordinate's accuracy. The errors that have a source in the incidence angle accuracy and roll accuracy grow the fastest with the incidence angle value. If the incidence angle is 0 (i.e., the laser beam is perpendicular to the scanned surface), the roll and incidence angle errors do not affect the $\mathrm{z}$ accuracy. According to the graph, the range influence decreases with a growing incidence angle value.

\subsection{Error analysis for existing ULS solutions}

After presenting the factors' influences separately, the entire impact on the point position is analysed using example combinations of laser scanner, GNSS/IMU unit and GNSS technique. Such examples can show the potential of theoretical analysis to predict errors that can occur in practice. Therefore, three combinations of a LiDAR module and a GNSS/INS module are considered, and the entire influence on the vertical and horizontal positions is examined. Additionally, the total error dependence on the operating altitude is analysed. Therefore, three different altitude values have been chosen $(40 \mathrm{~m}, 60 \mathrm{~m}$ and 80 $\mathrm{m})$. The accuracy characteristics of the selected modules have already been described and presented in Tables 1,2 (in appendix) and 3 (in appendix). Beyond the factors presented in Fig. 1, the GNSS accuracy is also included.

According to the graphs presented in Fig. 2, the most noticeable things are the differences in the vertical and horizontal accuracies. The total $\mathrm{x}$ and $\mathrm{y}$ error is higher than the $\mathrm{z}$ error for all combinations. Moreover, the differences grow slightly with the incidence angle because the horizontal accuracy (mxy) decreases faster. In addition, the GNSS error is also included in the total accuracy. In the combinations different types of GNSS positioning techniques are considered (RTK, PPP and DGPS). Depending on the measurement technique, the positioning error value varies. The DGPS accuracy is the lowest, which results in big differences between the selected module combinations, while the RTK and PPP methods are comparable.

In Fig. 3 the vertical and horizontal error distribution can be analysed. However, the influence of the individual factors cannot be seen. Therefore, accumulated graphs for three different incidence angle values - 0,30 and 60 degrees - have been created (Fig. 3). The same combinations of LiDAR and navigational modules as in the previous graphs have been selected. Due to the high differences in GNSS positioning error for the selected navigational modules, they are excluded in this part. Thanks to this, the proportions between the individual factors can be noticed more distinctly. The assumed flight height is equal to $40 \mathrm{~m}$.
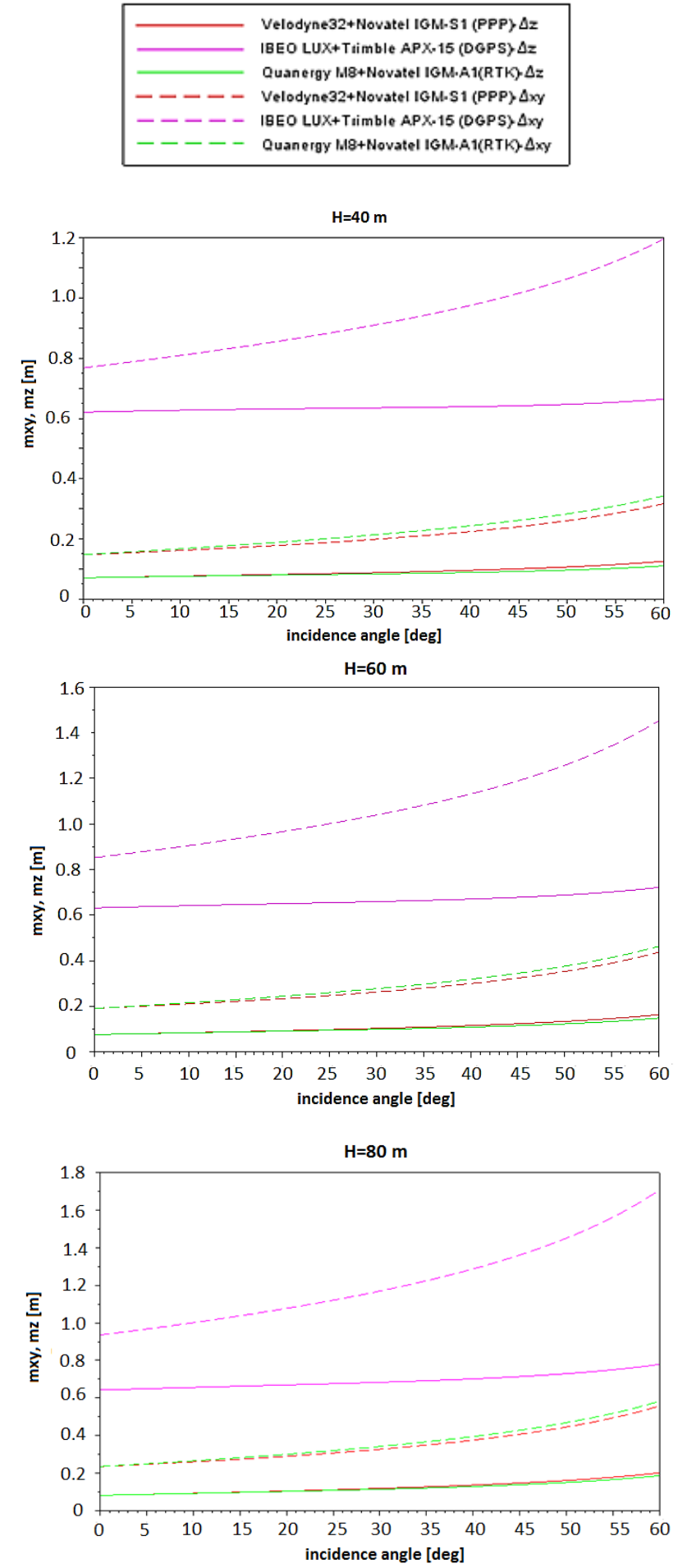

Figure 2. Graphs presenting the horizontal and vertical measurement accuracies for chosen combinations of LiDAR and navigational modules

According to the graphs presented in Fig. 3, the total accuracy, both horizontal and vertical, decreases with a growing incidence angle. The yaw and range influences on the horizontal error are especially distinctive. Based on the graphs, the horizontal error grows slightly faster than the vertical one. What is more, with a growing incidence angle value, the number of factors affecting the vertical accuracy also increases. For an incidence angle equal to 0 , only pitch and range affect the $\mathrm{z}$ accuracy, but if the incidence angle is 60 degrees, other factors show an influence (i.e., roll and theta). 

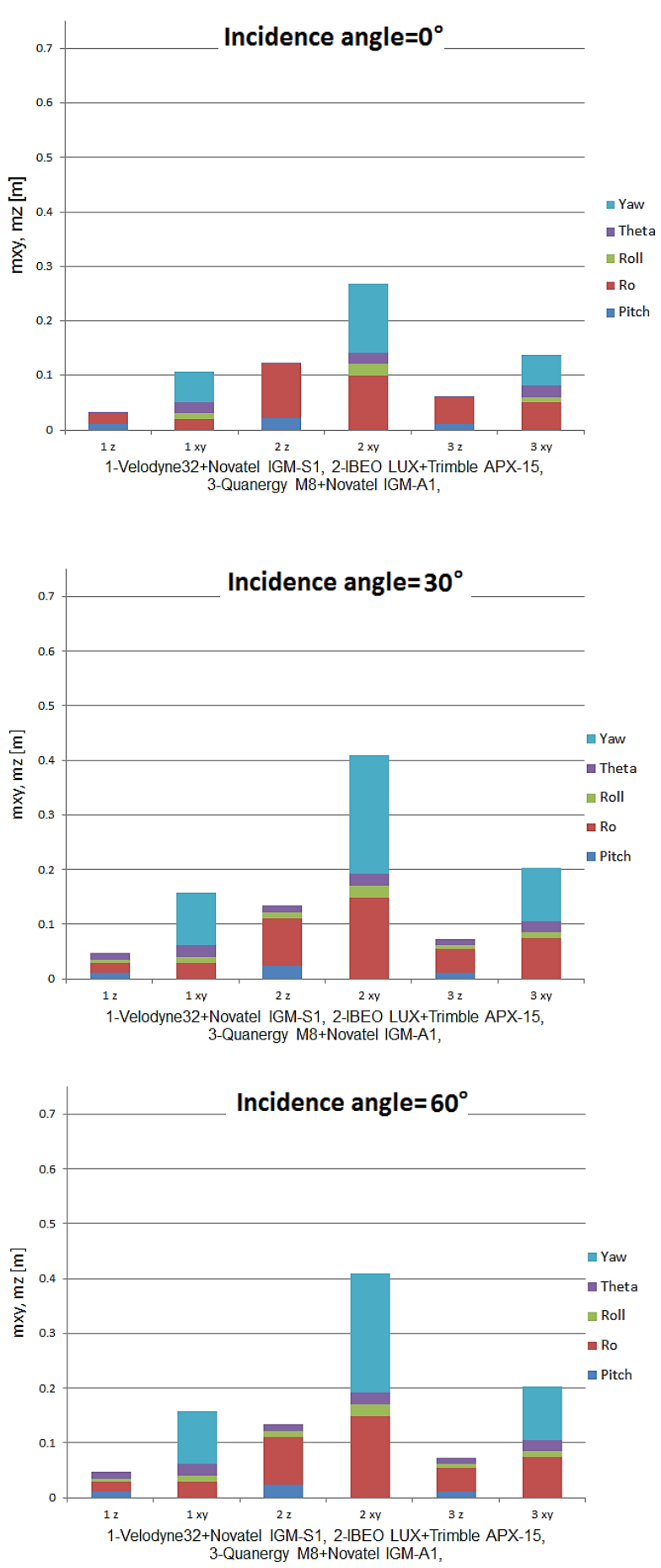

Figure 3. Accumulated graphs for yaw, pitch, roll, range and theta influence on horizontal and vertical position for 3 incidence angle values: 0,30 and 60 degrees (H=40 m, GNSS errors are excluded)

\subsection{Visualisation of error influence on final accuracy using experimental data}

The graphs that present the results of the calculations based on the mathematical formula can only illustrate how the theoretical measurement accuracy changes concerning various factors (e.g., roll, pitch or yaw) and how these values change with the incidence angle. However, it is hard to imagine how the 3D point position can be affected by these factors. Therefore, in this part of the article, a 3D visualisation of roll, pitch, yaw, theta and range errors' influence based on the transverse cross section of a point cloud is presented.
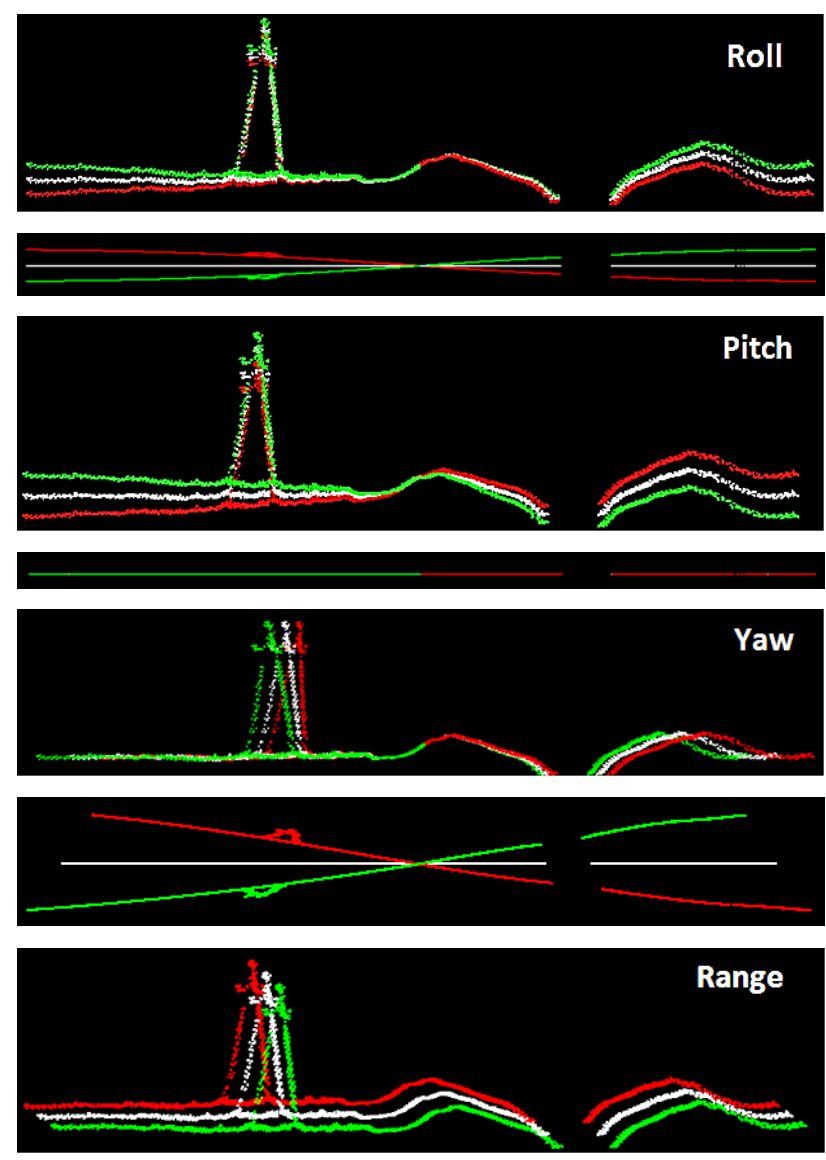

$\overline{\underline{+2}}$

Figure 4. Influence of roll, pitch, yaw and range errors on the point positions (white - reference point cloud, red-negative error value, green - positive error value)

In order to show how the factors influence a point cloud, a transverse cross section is used. This part of the point cloud presents an area near a river. The cross section contains an electricity pole and levees. Every factor is analysed separately in order to understand their impacts better. Additionally, the cross section is presented in two views: a top view and a front view. On every figure three cross sections are shown. The white point cloud is the reference one, green point represent a positive error value and red ones represent a negative error value. To show the impact of these errors, their sizes have been magnified for roll, pitch, yaw and range $(\mathrm{dRoll}=\mathrm{dPitch}=2$ degrees, $\mathrm{d}$ Yaw $=5$ degrees, $\mathrm{dRo}=1 \mathrm{~m})$.

As can be seen in Fig. 4, the influence of the factors is noticeable. First, the roll error influence is shown. Assuming the scanner position is in the middle of the cross section, the error influence increases with the scanning angle. A positive error value causes the height of the point to be overestimated. A similar result occurs when the error value is negative; in that case, the height value is underestimated. Pitch error causes height changes with the incidence angle; however, it is not a mirror reflection, as for the roll angle, but on one side the height is underestimated while on the other it is overestimated, depending on the error value. Concerning the yaw influence, the cross section is stretched or shortened, which can be easily noticed on the levee and on the electricity pole. The height values stay constant. Including the 
range error causes a cross section to be shifted to the right or to the left, depending on the error value, and in addition the height changes. Moreover, analysing the levees that are visible in the cross section, the height differences on the right slope are lower than on the left slope. For flat surfaces the vertical discrepancy is constant. Comparing the top views above, it can be seen that for every factor beyond the pitch, a rotation toward the vertical axis is noticed.

\section{DISCUSSION}

In order to analyse if the mathematical model can be applied properly to estimate the ULS accuracy, the results obtained by Jozkow et al. (2016), Bakuła et al. (2016) and Gallay et al. (2016) are discussed. It is worth highlighting that these articles were published in 2016, which shows the recently growing interest in light laser scanners. The error values of the laser scanners and IMU modules that were applied in the experiments in those publications are included in the mathematical model here.

According to Jozkow et al. (2016), the distance between the point cloud from the Nikon images and the point cloud from Velodyne was examined. The average distance value was $0.90 \mathrm{~m}$. According to the accuracy resulting from the mathematical model, after considering the components errors used by Jozkow et al., is up to $2 \mathrm{~m}$. A quite big difference between the mathematical model used in this article and experimental results obtained by Bakuła et al. (2016) is noticed. In this case, according to the mathematical model, the measurement accuracy could even reach $0.20 \mathrm{~m}$, contrary to the 0.09 obtained in Bakuła et al. experiment. In the last example (Gallay et al., 2016), the most accurate modules, both laser scanner and GNSS/IMU, were used. The horizontal accuracy of the post-processed flight was $0.020 \mathrm{~m}$ while the vertical was $0.025 \mathrm{~m}$. Based on the mathematical model, both the positional and vertical accuracies are approximately $0.035 \mathrm{~m}$.

According to the differences in the accuracies between the aforementioned experiments and the present mathematical model, the accuracies obtained in practice are better than the theoretical ones. The reason may be found in the model used in this article. The model is dedicated to airborne laser scanners, for which, for example, the IMU accuracy is higher than the IMU modules mounted on a UAV. Furthermore, the more accurate the IMU used in UAV laser scanning, the lower the difference between the mathematical model and the experimental results, as can be seen with Gallay et al.'s results. Thus, this issue may require further comparisons with experimental results. The theoretical model may require some modifications in order to be used in light laser scanning accuracy estimation.

\section{CONCLUSION}

Theoretical considerations on the basis of practical data are particularly important when planning to purchase and usage of a lightweight UAV laser scanning system. They are also important when creating scanning systems and selecting appropriate components for integration. Such an analysis can define the potential accuracy and future application of such a system. However, it should be noted that theoretical accuracy determined empirically does not always translate to the final accuracy of the data, which is also related to other conditions: weather influence, characteristics of the scanned area and, finally, the methodology for data processing.

Analysis of the mathematical model used in this paper confirms that the most important component of errors for scanning systems dedicated to UAVs is IMU units. These units do not need to be as accurate as the INS systems for airborne laser scanning systems because of the significantly lower flight altitude and, thus, a lower impact of angular measurement error on the accuracy of the final data. The high impact of the horizontal accuracy shows a yaw angle. On the basis of the calculations, it can also be seen that the influence of the pitch angle is negligible in comparison with other factors.

Analysing the solutions available on the market, it is worth noting that UAV laser scanners are mostly light, and it is easier to find ultralight laser scanners, whose weight is up to $2-3 \mathrm{~kg}$. The accuracy of these sensors is lower compared to other solutions (e.g., Riegl scanners). However, properly integrated modules, especially IMU modules, may result in new ultralight UAV scanners, which could be an alternative for both airborne and low-altitude solutions. To make UAV laser scanning comparable to the theoretical ALS accuracy, the applied GNSS receivers need to have comparable accuracy. The processing method needs to be similar, and the recommended techniques are RTK and PPP. The IMU solutions used in UAVs should be light, and their measurement accuracy can be 5-10 times lower for roll and pitch angles and up to 15 times lower for yaw than the IMU accuracy used in ALS.

\section{ACKNOWLEDGEMENTS}

Research financed by the National Centre for Research and Development in Defence, Security Programme, within the project "Advanced technologies in the prevention of flood hazard" (SAFEDAM).

\section{REFERENCES}

Bakuła, K., Ostrowski, W., Szender, M., Plutecki, W., Salach, A., Górski, K., 2016. Possibilities for Using LIDAR and Photogrammetric Data Obtained with AN Unmanned Aerial Vehicle for Levee Monitoring. The International Archives of the Photogrammetry, Remote Sensing and Spatial Information Sciences, XLI-B1.

Baltsavias, E. P., 1999. Airborne laser scanning: basic relations and formulas. ISPRS Journal of Photogrammetry \& Remote Sensing 54_1999. 199-214.

Colomina, I., Molina, P., 2014. Unmanned aerial systems for photogrammetry and remote sensing: A review. ISPRS Journal of Photogrammetry and Remote Sensing, 92, 79-97. http://doi.org/10.1016/j.isprsjprs.2014.02.013.

Gallay, M., Eck, C., Zgraggen, C., Kanuk, J., Dvorný, E., 2016. High Resolution Airborne Laser Scanning and Hyperspectral Imaging with a Small Uav Platform. ISPRS-International Archives of the Photogrammetry, Remote Sensing and Spatial Information Sciences, 823-827.

Glennie, C. L., Kusari, A., Facchin, A., 2016. Calibration and stability analysis of the VLP-16 laser scanner. ISPRS International Archives of the Photogrammetry, Remote Sensing and Spatial Information Sciences, XL(1), 10-12. http://doi.org/10.5194/isprsarchives-XL-3-W4-55-2016

Glennie, C., Lichti, D. D., 2010. Static Calibration and Analysis of the Velodyne HDL-64E S2 for High Accuracy Mobile Scanning. Remote Sensing 2010, 2, 1610-1624. 
Jozkow, G., Toth, C., Grejner-Brzezinska, D., 2016. UAS Topographic Mapping with Velodyne LiDAR Sensor. ISPRS Annals of the Photogrammetry, Remote Sensing and Spatial Information Sciences, Volume III-1, 201-208.

Li, Z., Yan, Y., Jing, Y., Zhao, S.G., 2015. The Design and Testing of a LiDAR Platform for a UAV for Heritage Mapping. The International Archives of the Photogrammetry, Remote Sensing and Spatial Information Sciences, Volume XL-1/W4.

Mandlburger, G., Pfennigbauer, M., Wieser, M., Riegl, U., Pfeifer, N., 2016. Evaluation of a Novel Uav-Borne TopoBathymetric Laser Profiler. ISPRS-International Archives of the Photogrammetry, Remote Sensing and Spatial Information Sciences, 933-939.

Mitteta, M.-A., Nouira, H., Roynard, X., Goulette, F., Deschaud, J.-E., 2016. Experimental Assessment of the Quanergy M8 LIDAR Sensor. ISPRS - International Archives of the Photogrammetry, Remote Sensing and Spatial Information Sciences, XLI(July), 527-531.

http://doi.org/10.5194/isprsarchives-XLI-B5-527-2016

Petrie, G., 2013. Current developments in airborne laser scanners suitable for use on lightweight UAVs: Progress is being made! GeoInformatics, 16(8), 16-22.
Schaer, P., Skaloud, J., Landtwing, S., Legat, K., 2007. Accuracy Estimation for Laser Point Cloud Including Scanning Geometry. Mobile Mapping Symposium 2007, Padova.

Sharma, A., Kumar, P., Ratnaker, S. M., Talole, S E., 2011. Accurate Navigation of UAV using Kalman filter based GPS/INS integration. Paper No. 208, in Proceedings of the 5th Symposium on Applied Aerodynamics and Design of Aerospace Vehicles, Bangalore.

Skaloud, J., Lichti, D., 2006. Rigorous approach to bore-sight self-calibration in airborne laser scanning. ISPRS Journal of Photogrammetry \& Remote Sensing 61, 47-59.

Starek, M., Jung, J., 2015. Lidar's Next Geospatial Frontier. GIM International, UAS Special, 25-27.

Vosselman, G., Maas, H-G., 2010. Airborne and Terrestrial Laser Scanning, chapter 3. Whittles Publishing.

Wang, Z., Shu, R., Xu, W., Pu, H., Yao, B., 2008. Analysis and recovery of systematic errors in airborne laser system. International Archives of the Photogrammetry, Remote Sensing and Spatial Information Sciences, XXXVII (B1): 289-294.

\section{APPENDIX}

Table 2 UAV LiDAR systems specifications

\begin{tabular}{|l|l|l|l|l|l|l|}
\hline Scanner model & LiDAR sensor & GNSS type & GNSS accuracy & $\begin{array}{l}\text { Pitch/Roll } \\
\text { accuracy }\end{array}$ & Heading accuracy & Weight \\
\hline Routescene LidarPod & HDL-32e & L1, Dual antenna & $0.8 / 1.5 \mathrm{~cm}$ & $0.15^{\circ}$ & $0.07^{\circ}$ & $2,50 \mathrm{~kg}$ \\
\hline Phoenix AL3-32 & HDL-32e & L1/L2, Dual antenna & $1 \mathrm{~cm}+1 \mathrm{ppm}$ & $0.015^{\circ}$ & $0.08^{\circ}$ & $3,20 \mathrm{~kg}$ \\
\hline Phoenix AL3-16 & VLP-16 & L1/L2, Dual antenna & $1 \mathrm{~cm}+1 \mathrm{ppm}$ & $0.015^{\circ}$ & $0.08^{\circ}$ & $2,50 \mathrm{~kg}$ \\
\hline Phoenix Scout & VLP-16 & L1/L2, Dual antenna & $1 \mathrm{~cm}+1 \mathrm{ppm}$ & $0.015^{\circ}$ & $0.08^{\circ}$ & $1,85 \mathrm{~kg}$ \\
\hline YellowScanSurveyor & VLP-16 & L1/L2, Single antenna & $2-5 \mathrm{~cm}$ & $0.03^{\circ}$ & $0.18^{\circ} / 0.08^{\circ}$ & $1,50 \mathrm{~kg}$ \\
\hline LidarUSA Snoopy & HDL-32e & L1/L2, Single antenna & $1-23 \mathrm{~cm}$ & $0.017^{\circ}$ & $0.08^{\circ}$ & $2,50 \mathrm{~kg}$ \\
\hline YellowScan Mapper & IBEO LUX & L1/L2, Single antenna & $?$ & $?$ & $?$ & $2,10 \mathrm{~kg}$ \\
\hline LidarUSA ScanLook & Quanergy M8 & L1, Dual antenna & $2.5 \mathrm{~m}$ & $0.10^{\circ}$ & $0.30^{\circ}$ & $1,50 \mathrm{~kg}$ \\
\hline Sabre Sky-3D S80 & $\begin{array}{l}\text { Hokuyo } \\
\text { UXM- }\end{array}$ & L1/L2, Dual antenna & 0.025 & $0.025^{\circ}$ & $0.06^{\circ}$ & $4.98 \mathrm{~kg}$ \\
\hline SabX-EWA & FARO & L1/L2, Dual antenna & $0.02-0.05$ & $0.025^{\circ}$ & $0.06^{\circ}$ & $0.015^{\circ}$ \\
\hline
\end{tabular}


Table 3. Light UAV GNSS/IMU modules specifications

\begin{tabular}{|c|c|c|c|c|c|c|c|c|}
\hline & $\begin{array}{l}\text { VectorNav } \\
\text { VN-300 }\end{array}$ & $\begin{array}{l}\text { VectorNav } \\
\text { VN-200 }\end{array}$ & $\begin{array}{l}\text { Novatel } \\
\text { IGM-A1 }\end{array}$ & $\begin{array}{l}\text { Novatel } \\
\text { IGM-S1 }\end{array}$ & $\begin{array}{l}\text { Trimble } \\
\text { APX-15 }\end{array}$ & $\begin{array}{l}\text { SBG } \\
\text { Systems } \\
\text { Ekinox-N }\end{array}$ & $\begin{array}{l}\text { SBG } \\
\text { Systems } \\
\text { Ellipse-N }\end{array}$ & $\begin{array}{l}\text { Microstrain } \\
\text { 3DM-RQ1 }\end{array}$ \\
\hline GNSS type & $\begin{array}{l}\text { L1 GNSS. } \\
\text { Dual } \\
\text { Antenna }\end{array}$ & $\begin{array}{l}\text { L1 GNSS. } \\
\text { Single } \\
\text { Antenna }\end{array}$ & $\begin{array}{l}\text { L1/L2 GPS. } \\
\text { Single } \\
\text { Antenna }\end{array}$ & $\begin{array}{l}\text { L1/L2 GPS. } \\
\text { Single } \\
\text { Antenna }\end{array}$ & $\begin{array}{l}\text { L1/L2. } \\
\text { Single } \\
\text { Antenna }\end{array}$ & $\begin{array}{l}\text { L1/L2 } \\
\text { GNSS. } \\
\text { Single } \\
\text { Antenna }\end{array}$ & $\begin{array}{l}\text { L1 GNSS. } \\
\text { Single } \\
\text { Antenna }\end{array}$ & $\begin{array}{l}\text { L1. GPS. } \\
\text { Single } \\
\text { Antenna }\end{array}$ \\
\hline $\begin{array}{l}\text { Position RMS } \\
\text { [m] }\end{array}$ & 2.5 & 2.5 & $\begin{array}{l}0.4 \text { (DGPS). } \\
0.01+1 \mathrm{ppm} \\
\text { (RTK) }\end{array}$ & $\begin{array}{l}0.4 \text { (DGPS). } \\
0.04 \text { (PPP). } \\
0.01+1 \mathrm{ppm} \\
\text { (RTK) }\end{array}$ & $\begin{array}{l}0.5-2 \\
\text { (DGPS). } \\
0.02-0.05 \\
\text { (RTK) }\end{array}$ & $\begin{array}{l}0.4 \text { (DGPS) } \\
.0 .02 \\
\text { (RTK/PPP) }\end{array}$ & 2 & 2.5 \\
\hline $\begin{array}{l}\text { Roll \& Pitch } \\
\text { Accuracy }\end{array}$ & $0.1^{\circ}$ & $0.1^{\circ}$ & $\begin{array}{l}0.015 \text { to } \\
0.017^{\circ}\end{array}$ & $\begin{array}{l}0.015 \text { to } \\
0.017^{\circ}\end{array}$ & $0.03^{\circ}$ & $\begin{array}{l}0.05^{\circ}- \\
0.02^{\circ}\end{array}$ & $0.2^{\circ}$ & $0.1^{\circ}$ \\
\hline $\begin{array}{l}\text { Heading } \\
\text { Accuracy }\end{array}$ & $\begin{array}{l}0.3^{\circ} \\
\text { (dynamic) }\end{array}$ & $\begin{array}{l}0.3^{\circ} \\
\text { (dynamic) }\end{array}$ & $0.08^{\circ}$ & $0.08^{\circ}$ & $0.18^{\circ}$ & $0.1-0.04^{\circ}$ & $0.5^{\circ}$ & $0.5^{\circ}$ \\
\hline $\begin{array}{l}\text { Gyroscope } \\
\text { range }\end{array}$ & $\pm 2000 \% \mathrm{~s}$ & $\pm 2000 \% / \mathrm{s}$ & $\pm 450^{\circ} / \mathrm{sec}$ & $\pm 400^{\circ} / \mathrm{sec}$ & $?$ & $\pm 400^{\circ} / \mathrm{sec}$ & $\pm 400^{\circ} / \mathrm{sec}$ & $\pm 900^{\circ} / \mathrm{sec}$ \\
\hline $\begin{array}{l}\text { Gyroscope } \\
\text { bias stability }\end{array}$ & $<10^{\circ} / \mathrm{hr}$ & $<10^{\circ} / \mathrm{hr}$ & $6^{\circ} / \mathrm{h}$ & $0.5^{\circ} / \mathrm{h}$ & $?$ & $<3 \% \mathrm{hr}$ & $8^{\circ} / \mathrm{h}$ & $5^{\circ} / \mathrm{h}$ \\
\hline Weight & $30 \mathrm{~g}$ & $16 \mathrm{~g}$ & $515 \mathrm{~g}$ & $540 \mathrm{~g}$ & $60 \mathrm{~g}$ & $500 \mathrm{~g}$ & $47 \mathrm{~g}$ & $205 \mathrm{~g}$ \\
\hline
\end{tabular}

